\title{
Preclinical and Coclinical Studies in Prostate Cancer
}

\author{
Ming Chen and Pier Paolo Pandolfi \\ Cancer Research Institute, Beth Israel Deaconess Cancer Center, Department of Medicine and Pathology, \\ Beth Israel Deaconess Medical Center, Harvard Medical School, Boston, Massachusetts 02215 \\ Correspondence: ppandolf@bidmc.harvard.edu
}

Men who develop metastatic castration-resistant prostate cancer (mCRPC) will invariably succumb to their disease. Thus there remains a pressing need for preclinical testing of new drugs and drug combinations for late-stage prostate cancer (PCa). Insights from the mCRPC genomic landscape have revealed that, in addition to sustained androgen receptor (AR) signaling, there are other actionable molecular alterations and distinct molecular subclasses of PCa; however, the rate at which this knowledge translates into patient care via current preclinical testing is painfully slow and inefficient. Here, we will highlight the issues involved and discuss a new translational platform, "the co-clinical trial project," to expedite current preclinical studies and optimize clinical trial and experimental drug testing. With this platform, in vivo preclinical and early clinical studies are closely aligned, enabling in vivo testing of drugs using genetically engineered mouse models (GEMMs) in defined genetic contexts to personalize individual therapies. We will discuss the principles and essential components of this novel paradigm, representative success stories and future therapeutic options for mCRPC that should be explored.

P ostate cancer $(\mathrm{PCa})$ is the third leading cause of cancer-related deaths in U.S. men. Although localized PCa is highly curable, metastatic castration-resistant PCa (mCRPC) is still a fatal disease (Wu et al. 2014). Preclinical studies implicating dysregulated androgen receptor (AR) signaling as the predominant driver of mCRPC have propelled the development of novel agents targeting the AR signaling (Chen et al. 2004). Despite the effectiveness of recently approved AR-targeted therapies, such as abiraterone and enzalutamide, de novo or acquired drug resistance invariably emerge and pose the largest obstacle to implementing curable thera- py (Watson et al. 2015). Thus, there remains a pressing need for preclinical testing of new drugs and novel drug combinations that are tailored to target potent driver mutations and prevent resistance in $\mathrm{MCRPC}$.

Integrative genomic analyses have shown that PCa displays significant inter- and intrapatient heterogeneity, not only in the context of the mutational landscape but also in epigenetic deregulation and aberrant transcriptome of noncoding elements (Boutros et al. 2015; Cooper et al. 2015; Gundem et al. 2015). It is now widely understood that defining the genetic makeup of individual patient tumors is likely to have a sub-

Editors: Michael M. Shen and Mark A. Rubin

Additional Perspectives on Prostate Cancer available at www.perspectivesinmedicine.org

Copyright (C) 2018 Cold Spring Harbor Laboratory Press; all rights reserved; doi: 10.1101/cshperspect.a030544

Cite this article as Cold Spring Harb Perspect Med 2018;8:a030544 
stantial impact on the effectiveness of their treatment, a concept referred to as precision medicine (Collins and Varmus 2015). The recent development of powerful methods for characterizing patients, particularly high-throughput "omics" analysis, and large-scale biologic databases are now enabling researchers to categorize and stratify patients according to genetic alterations, and in doing so, to distinguish different subsets of human PCa, how these genetic makeups dictate tumor aggressiveness, and likely response to the standard-of-care (SOC) and/or experimental therapies, ultimately leading to precise and personalized therapies. Indeed, stratification of patients with defined genetic defects has recently shown great promise for targeted therapies in PCa (Mateo et al. 2015). However, this opportunity is not without its own challenges, particularly concerning the design of clinical trials. With increased PCa diversity and complexity, one major hurdle is the stark decrease in the size of patient populations available for enrollment in appropriate clinical trials. This, in turn, makes it difficult to obtain robust statistical analyses, and prolongs the time that it takes new and/or combinatorial therapies to be evaluated.

In recognition of these challenges, we are now compelled to rethink the platforms that are required to rapidly analyze in model systems current and novel therapeutic options for patients to ensure rapid implementation of personalized approaches. Preclinical studies using accurate genetically engineered mouse models (GEMMs) of specific cancer subtypes have proven extremely valuable; indeed, they have already guided and optimized the treatment of several forms of human cancer (Van Dyke and Jacks 2002; Abate-Shen and Pandolfi 2013). However, the traditional "GEMMs-to-human" or "preclinical-to-clinical" approach remains painfully slow because data have to be accrued in the mouse model of interest first, and then translated to be able to inform the clinical trials that follow. Importantly, current preclinical methods to predict successful outcomes are extremely limited because only about $5 \%$ of new cancer drugs are approved after preclinical studies (Sharpless and Depinho 2006). Given that we are able to model cancer in mice at an unprecedented level of accuracy and that a large amount of new experimental drugs are currently in earlyphase clinical studies, we have implemented a coclinical trial project to align preclinical efforts in GEMMs with clinical trial efforts in humans to optimize and refine phase I/II clinical studies in real time and expedite effective drug approval for personalized therapies.

In this review, we discuss the principles and essential components of the coclinical trial project, with a focus on how the implementation of such a platform can accelerate the development of personalized medicine for PCa. We also summarize what we have learned thus far by using this platform and future therapeutic options for mCRPC that have emerged, and should be explored, on the basis of these efforts.

\section{THE COCLINICAL TRIAL PROJECT FOR THE DEVELOPMENT OF PERSONALIZED THERAPIES}

\section{The Approach}

The coclinical trial project rests on the notion that (1) cancer develops in many distinct genetic subtypes, (2) this diversity impacts how these tumors respond to or resist various forms of therapy, and (3) that preclinical testing of new drugs and drug combinations in faithful GEMMs of human cancer is a powerful and predictive platform for clinical trial optimization. The prospect of implementing this approach has been significantly improved by the advance of powerful "omics" methods for characterizing patients and our ability to model cancer in mice at an unprecedented level of accuracy. The keys to this approach are "synchronicity" and "integration." Specifically, by enrolling as many GEMMs representative of the genetic diversity of a given tumor type as in which human trial is performed, and treating GEMMs and human patients in exactly the same way, the relevant clinical, biological, and pharmacologic data (i.e., sensitivity vs. resistance to specific regimens, imaging analysis, microarray and proteomic profiling, somatic mutation, nucleotide polymorphisms, etc.) from both sources are an- 
Pre- and Coclinical Studies in Prostate Cancer

alyzed in parallel, shared, and integrated in real time. The synchronicity and the integration of the approach in turn credential and validate, in real time, what is found in human and mice, thereby facilitating the design of subsequent clinical studies through more timely and informed clinical decisions (Fig. 1).

The coclinical trial project can significantly lessen the gap between preclinical study and patient care via (1) the rapid stratification of patient sensitivity and resistance to a specific treatment on the basis of molecular and genetic criteria, (2) characterization of mechanisms dictating de novo or acquired tumor resistance to therapy, and (3) the use of this platform as a testing ground for new drug combinations (Fig. 1). Instead of attempting to provide a detailed review for the coclinical trial project, here we will focus on the essential components rele- vant to PCa and refer the reader to previous articles that have addressed specific topics and/ or cancer types in depth (Nardella et al. 2011; Chen et al. 2012; Lunardi et al. 2013; Kwong et al. 2015; Whitley et al. 2016).

\section{GEMMs as in Vivo Models to Inform Patient Stratification}

Mice rarely develop spontaneous PCa; however, they can be genetically or environmentally modified to develop PCa, and are a compelling model system for the in vivo testing of new drugs and drug combinations. Implant of cancer cell lines (xenografts) or patient-derived xenografts (PDXs) into heterologous sites of immunodeficient mice represent additional ways to study human cancer in mouse models. These models can also be used "coclinically." However, cell

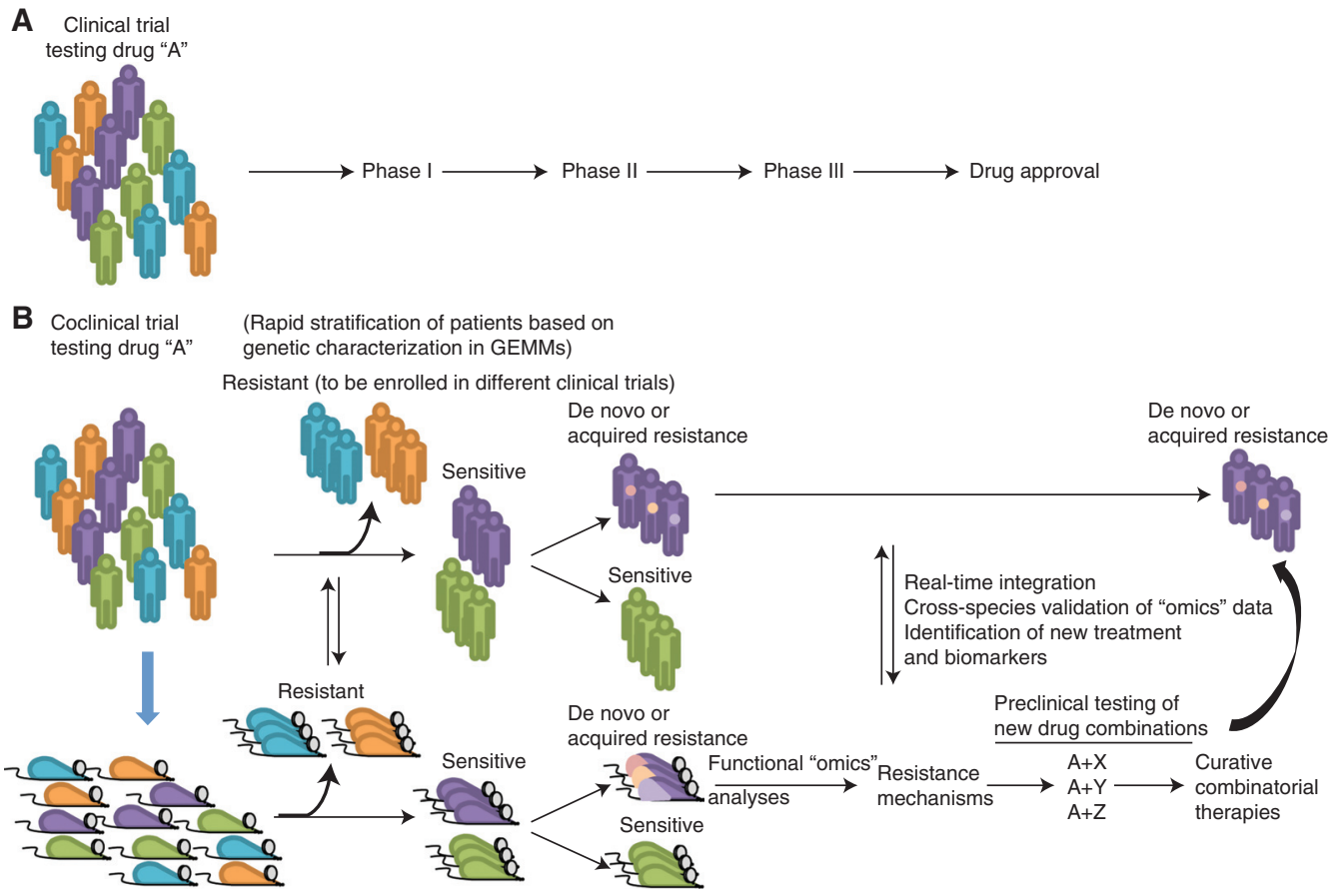

Figure 1. The coclinical trial platform. (A) In the traditional clinical trial, little attention is paid to the molecular characteristics of the disease. When a minor fraction of patients are responsive, the overall response of the population might mask the responders. $(B)$ In the coclinical trial project, relevant genetically engineered mouse models (GEMMs) and human patients are treated with the same drug and clinical protocol. Integrated analyses of data accrued in GEMMs and patients serve to stratify responsiveness and resistance on the basis of molecular and genetic criteria. Mechanisms underlying acquired resistance are also rapidly identified, and drug combinations to overcome such resistance are tested in GEMMs for their effectiveness. 
lines or PDXs do harbor multiple concurrent genetic alterations at once, which makes it difficult to determine the impact of specific mutations in modulating drug or drug combination response. Additionally, these models are immunodeficient and hence not amenable to test cancer immunotherapies. For more information on these topics, we refer you to Risbridger et al. (2017). Central to the coclinical trial project are faithful GEMMs to steer parallel clinical studies in patients. There are numerous advantages in using GEMMs coclinically. First, GEMMs develop tumors de novo in the context of the native tissue environment and intact immune system. This criterion is paramount for the study of cancer treatment, particularly when considering the role of the tumor stroma in resistance to therapy and the potential for use of immunotherapy in the treatment of PCa (Kantoff et al. 2010; Boelens et al. 2014). Second, cancer phenotypes in GEMMs arise from well-defined genetic alterations that have been known to faithfully recapitulate human cancer. For example, many GEMMs of PCa are based on loss of function of Pten, which is among the most frequently lost or mutated tumor suppressors in human PCa (Taylor et al. 2010; Barbieri et al. 2012; Grasso et al. 2012; Robinson et al. 2015). Moreover, the specific genetic alterations enable us to simplify and deconstruct the precise genetic elements underlying resistance or sensitivity to a given treatment modality. Third, GEMMs enable both longitudinal and molecular analyses of cancerous lesions that can lead to identification of novel biomarkers of response and facilitate formulation of new combinatorial therapeutic modalities. Fourth, GEMMs can be readily expanded to enable more subjects, drugs, and drug combinations to be tested in the coclinical setting. This has shown to be of great value, in particular to the study of genetically rare cancer subtypes of which sizable patient accrual represents a major hurdle.

Like other types of cancer, $\mathrm{PCa}$ is characterized by a wide range of genetic complexity, such as loss and mutation of tumor suppressor genes (e.g., PTEN, NKX3.1, P53, SPOP, SMAD4, LRF, $P L Z F$, and $B R C A 1 / 2$ ), amplification and mutation of oncogenes (e.g., $c-M Y C, P I 3 K C A / B$, and
$A R$ ), and fusion genes (e.g., ERG/ETV1/ETV4). In recent years, this disheartening complexity has been successfully modeled in GEMMs (Irshad and Abate-Shen 2013), allowing for the generation of a unique genetic platform of proxy-PCa in the mouse, which has proven to be essential in elucidating the role of specific genetic perturbations in the onset and progression of human PCa. Importantly, substantial efforts have been devoted to systematically applying this resource in the preclinical/coclinical setting to test new drugs and drug combinations for targeted therapies, to dissect the mechanisms underlying de novo or acquired tumor resistance to SOC treatments, and to discover new treatment combinations (Carver et al. 2011; Lunardi et al. 2013; Schwartz et al. 2015; Yamamoto et al. 2015). However, challenges still remain in the development of these models. Notably, PCa is made up of complex genetic landscapes, and we currently have a limited ability to model tumor heterogeneity. Moreover, metastasis has proven to be particularly challenging to model, and the progression to metastasis from either a primary indolent or advanced-stage disease is rarely observed for the majority of the GEMMs of PCa (Ittmann et al. 2013), but we can envisage that technological developments such as CRISPRCas9 (clustered regularly interspaced short palindromic repeats-CRISPR-associated 9) technology (Hsu et al. 2014) would facilitate modeling PCa genetic diversity, complexity, and metastasis in GEMMs and address these challenges. Despite the current limitations, the use of GEMMs is a powerful tool for both preclinical and coclinical evaluation and fine-tuning of treatment protocols in specific genetic makeups precisely for its ability to simplify and deconstruct the critical genetic elements dictating differential responses to treatment.

\section{Components and Requirements for the Coclinical Trial: The Mouse Hospital and Beyond}

The simultaneous execution of preclinical trials in mice and phase I/II trials in humans is incredibly resource-intensive (Fig. 2). To ensure the coclinical trial project success, a "mouse hospi- 


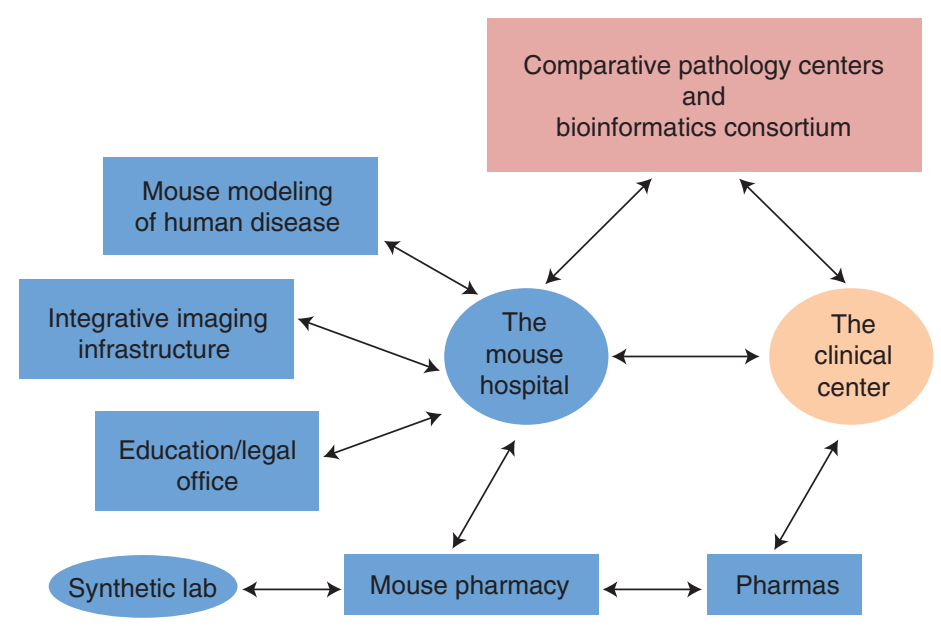

Figure 2. Fundamental components of the coclinical effort and their relationship to infrastructure and pharmaceutical companies. To ensure that preclinical and coclinical trials are most effective, mouse trials are performed in a manner and environment similar to those for human patients. Infrastructural and operational procedures need to be in place to ensure that data are readily transferable to the clinic. Acquisition of relevant therapeutic agents for coclinical evaluation is coordinated by the mouse pharmacy, which might obtain agents directly from industry or independently coordinated synthesis of relevant agents.

tal" with a state-of-the-art infrastructure for in vivo mouse imaging and a mouse pharmacy is required for a consistent and reproducible pipeline to be maintained. This hospital-type animal facility is staffed by experts in levels of mouse care (i.e., husbandry, treatments, and surgery) with access to procedure rooms and small animal in vivo-imaging technologies (bioluminescence, magnetic resonance imaging, microcomputed tomography, micro-positron emission tomography, ultrasonogram, fluorescencemediated tomography), as well as instruments needed for blood and urine tests. All of the above are fundamental to determining local and systemic tumor responses to treatments in GEMMs. Moreover, several other important elements are also required for integration and implementation of mouse-human coclinical trials (such as bioinformatics consortia, comparative pathology centers, and central databases). Even further, to maintain consistent workflows and generate reproducible and reliable high-quality results, a system of work needs to be established, ensuring that procedures, data collection, and informatics analyses are performed consistently throughout the mouse hospital and among mouse hospitals. This is essential to be able to share and compare mouse data across different mouse hospitals and human clinical centers. Most important, this platform requires a sustained and coordinated effort between basic scientists and clinicians (Fig. 2).

\section{The Coclinical Trial Efforts in PCa}

Proof-of-principle examples of the efficacy of the coclinical trial platform have been recently reported in $\mathrm{PCa}$ (Lunardi et al. 2013). The mainstay of therapy for advanced and metastatic $\mathrm{PCa}$ for more than 70 years has been androgen-deprivation therapy (ADT) (Huggins and Hodges 1941). Despite the majority of patients experiencing an initial positive response to $\mathrm{ADT}$, most tumors eventually acquire resistance to this treatment and, as a result, patients inevitably relapse to a lethal stage of the disease. However, there is a wide variability in the durability of ADT efficacy, with responses varying from months to years. Not much is known about the possible determinants of ADT response. Given the genetic heterogeneity of PCa, we have hypothesized that different genetic lesions driving the tumorigenic process may also affect ADT efficacy. 
Accordingly, in a coclinical trial effort, we have enrolled a cohort of 84 PCa patients and a panel of GEMMs bearing common human PCa genetics (Pten ${ }^{\mathrm{pc}-/-}$; Pten ${ }^{\mathrm{pc}-1} p 53^{\mathrm{pc}-/-}$; $\left.P t e n^{\mathrm{pc}-/} L r f^{\mathrm{pc}^{\mathrm{c}-l-}}\right)$, in which we rapidly identified Pten-p53 and Pten-Lrf double-null tumors in mice as resistant to ADT. We anticipated the response of patients with $\mathrm{PCa}$ characterized by the same genetic lesions using these coclinical data sets. Importantly, a comparative genetic and molecular analysis of Pten-null (ADT-sensitive) and Pten-p53 and Pten-Lrf double-null (ADT-resistant) mouse prostate tumors showed up-regulation of the steroid-5- $\alpha$ reductase type 1 gene (Srd5a1) and deregulation of the X-linked inhibitor of apoptosis protein (XIAP)-associated factor 1(Xaf1)/XIAP) apoptotic pathway as two potential mechanisms of ADT resistance. A double combination of embelin (a natural XIAP inhibitor) and dutasteride (an SRD5A1 inhibitor currently being studied in clinical trials for ADT-resistant PCa, ClinicalTrials.gov identifier: NCT01393730, NCT00673127, NCT00 553878 , NCT00403000), formally proved the functional role of deregulation of both pathways in ADT resistance by rescuing ADT efficacy in vivo in both Pten-p53 and Pten-Lrf double-null mouse prostate tumors and in vitro in human PCa cell lines. This, in turn, paved the way for the clinical testing of such a combinatorial approach, and of novel predictive biomarkers and therapeutic targets for $\mathrm{ADT}$ resistance in men. Coclinical efforts have by now also shown significant promise toward a real-time optimization of patient stratification in the effective treatment of several other types of cancers (Chen et al. 2012, 2014; Kwong et al. 2015; Whitley et al. 2016).

\section{TACKLING CHALLENGES AND OPPORTUNITIES FOR PRECISION MEDICINE IN PCa THROUGH COCLINICAL EFFORTS}

\section{The Clinical Challenges}

The survival of mCRPC patients has been improved substantially by the use of abiraterone and enzalutamide as single-agent therapies against the AR pathway (Scher et al. 2012; Ryan et al. 2013; Beer et al. 2014). Unfortunately, to date, no single agent has been able to cure PCa on its own, largely owing to tumor heterogeneity and the adaptive responses that support the development of de novo or acquired drug resistance. Another challenge is the relief of profound feedback inhibition of multiple oncogenic pathways triggered by anti-AR drugs that limit the success of targeted therapies (Chandarlapaty 2012). As such, it is therefore essential to be able to continue to identify additional effective strategies to combine with SOC treatment toward a curative regimen.

\section{Therapeutic Opportunities for Personalized Therapies in $\mathrm{mCRPC}$}

Recent mCRPC genomic landscape studies have identified multiple molecular alterations in other actionable pathways, aside from AR, that drive mCRPC progression (Fig. 3), including PI3K/AKT/PTEN, RAF, DNA repair, WNT, cell cycle, and cellular metabolism (Taylor et al. 2010; Grasso et al. 2012; Robinson et al. 2015). To this end, the coclinical trial using GEMMs is an ideal investigational platform to rapidly test drug combinations targeting these molecular lesions in different genetic makeups to ensure rapid implementation of personalized treatment. Here, we highlight future therapeutic opportunities for mCRPC that should be further explored through using the coclinical platform, and, for information regarding AR-targeted therapies, we refer you to Centenera et al. (2017).

The Phosphoinositide-3 Kinase (PI3K)/AKT/ Mechanistic Target of Rapamycin (mTOR) Pathway

The PI3K/AKT/mTOR pathway is altered in $\sim 50 \%$ of mCRPC through either PTEN inactivation or/and aberrant activation in PIK3CA/B (Taylor et al. 2010; Grasso et al. 2012; Robinson et al. 2015). Several studies have also shown cross talk between the AR pathway and PI3K signaling, in which these pathways regulate each other by reciprocal negative feedback, such that inhibition of one activates the other, 
Pre- and Coclinical Studies in Prostate Cancer

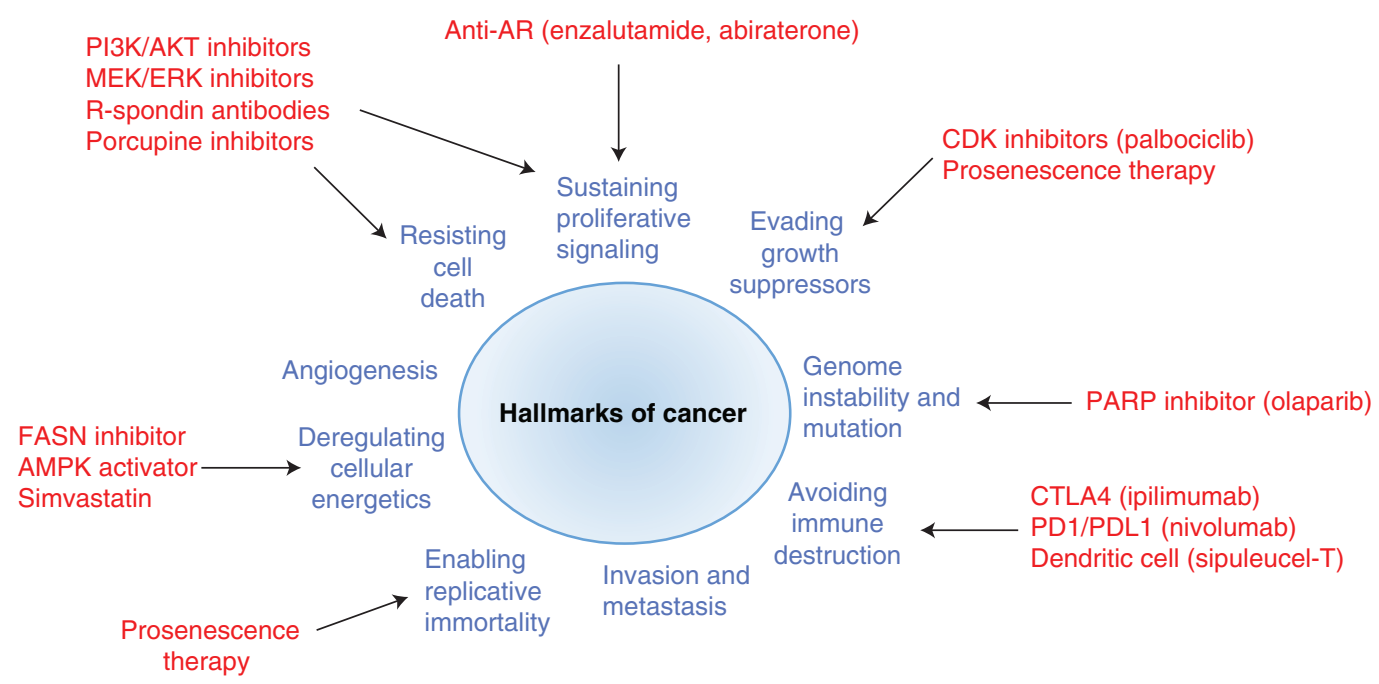

Figure 3. Therapeutic targets in metastatic castration-resistant prostate cancer (mCRPC). AR, Androgen receptor; CDK, cyclin-dependent kinase; PARP, poly(ADP-ribose) polymerase; AMPK, AMP-activated protein kinase; FASN, fatty acid synthase.

therefore providing rationale for combination therapy in mCRPC patients (Carver et al. 2011; Schwartz et al. 2015). Phase I/II trials assessing the combination of next-generation AR therapy with a PI3K/AKT/mTOR inhibitor are currently ongoing (ClinicalTrials.gov identifier: NCT02407054 and NCT02215096).

\section{Ras/Raf/MEK/ERK Mitogen-Activated Protein Kinase (MAPK) Pathway}

The MAPK signaling pathway is often found aberrantly activated in advanced metastatic PCa (Kinkade et al. 2008; Taylor et al. 2010). However, little is known about the underlying molecular mechanisms leading to MAPK activation, because activating mutations or gene rearrangements involving the MAPK cascade are rare in human PCa. Nevertheless, aberrant KRAS/B-RAF/RAF1 fusions have been identified in 3\% of $\mathrm{mCRPC}$, representing a rare subset of mCRPC (Palanisamy et al. 2010; Wang et al. 2011; Beltran et al. 2013; Robinson et al. 2015). Previous studies have shown that expression of RAF fusion protein in prostate cells induces a neoplastic phenotype that is sensitive to RAF and MEK inhibitor, suggesting that tumors from mCRPC patients harboring RAF fusion might be sensitive to pan-RAF inhibitors or MEK inhibitors (Palanisamy et al. 2010). Because the available cohort of MCRPC patients with RAS/RAF fusion is small, the coclinical trial platform with faithful GEMMs in this rare subtype of PCa will be able to better evaluate targeted combinatorial therapies. Notably, the PI3K/AKT/mTOR and ERK-MAPK signaling pathways have been shown to cooperate in PCa progression and contribute to the development of castration-resistant and metastatic $\mathrm{PCa}$ (Gao et al. 2006). On this basis, dual targeting of the PI3K/AKT/mTOR and ERK-MAPK signaling has been proven highly effective in suppressing $\mathrm{mCRPC}$ in preclinical studies (Kinkade et al. 2008; Floc'h et al. 2012; Toren et al. 2016).

\section{DNA Repair Pathway}

Another common subset of $\mathrm{mCRPC}$ is characterized by defects in DNA repair, accounting for $20 \%-30 \%$ of sporadic mCRPC, as exemplified by germline and somatic mutations in genes that encode proteins in DNA repair pathways, such as $B R C A 1 / 2$ and ataxia-telangiectasia mutated (ATM) (Grasso et al. 2012; Beltran et al. 2013; 
Robinson et al. 2015). BRCA-defective cells are unable to repair double-strand DNA breaks by homologous recombination, and thus become highly sensitive to inhibitors of the DNA repair enzyme, poly(ADP-ribose) polymerase (PARP), suggesting that treatment with a PARP inhibitor may exploit a synthetically lethal interaction. In a single-agent phase II trial, one such inhibitor, olaparib, led to a high response rate in $\mathrm{mCRPC}$ patients whose cancers were defective in DNA repair pathways (Mateo et al. 2015). Interestingly, not only the aberrations in DNA repair pathway, but also the overexpression of ETS, PTEN loss, or SPOP mutations have been associated with sensitivity to PARP inhibitor in human PCa (Brenner et al. 2011; Gonzalez-Billalabeitia et al. 2014; Boysen et al. 2015). Considering the potential use of olaparib in combinatorial therapies against various common genetic alterations in mCRPC, the coclinical platform should be used to explore responsiveness and sensitivity within distinct molecular subclasses of PCa (see the example of Gonzalez-Billalabeitia et al. 2014).

\section{Wnt Signaling}

Wnt signaling is one of the key oncogenic pathways in multiple types of cancer and is altered in $18 \%$ of mCRPC (Grasso et al. 2012; Robinson et al. 2015). Dysregulation of WNT signaling occurs through mutations of APC and CTNNB1, overexpression of Wnt ligands, such as $R$-spondin 2/3, R-spondin fusions, mutations of the Rspondin coreceptors RNF43/ZNFR3, or aberrant SOX9 activation. Recent studies have shown that cancer cells with aberrations in RNF43, ZNRF3, or RSPO2/3 (overall 5\% of mCRPC patients) or SOX9 overexpression, have a high response rate to the porcupine inhibitor (Liu et al. 2013; Ma et al. 2016), suggesting that a subset of mCRPC patients would benefit from Wnttargeted therapies. In addition, R-spondins are emerging as an important cancer target (Storm et al. 2016). Therefore, targeting the R-spondin pathway in mCRPC tumors harboring Wnt pathway alterations or specifically R-spondin fusions may be worthy of further investigations in preclinical and coclinical settings.

\section{Cell-Cycle Control and Prosenescence Therapy}

The cyclin/cyclin-dependent kinase (CDK)/retinoblastoma (RB) axis is a critical modulator of cell-cycle entry and is aberrant in more than $20 \%$ of mCRPC. Cell-cycle derangement, such as through CCND1 amplification or CDKN2A/ $\mathrm{B}$ loss, results in enhanced response to CDK4/6 inhibitors in preclinical mCRPC models (Comstock et al. 2013), suggesting that the selective CDK4/6 inhibition as a potential therapeutic regime in $\mathrm{mCRPC}$ warrants further examination to evaluate its clinical efficacy in a coclinical setting.

By modulating the cell-cycle machinery and status, prosenescence therapy for tumor prevention and suppression can also be developed. Cellular senescence has emerged as an intrinsic tumor-suppressive mechanism, which can be enhanced by stabilizing p53, Rb, p27, and induction of PTEN-loss-induced senescence (PICS) (Chen et al. 2005; Takahashi et al. 2006; Alimonti et al. 2010). Enhancing the prosenescence response in PCa tumors has had a substantial therapeutic effect in preclinical studies (Alimonti et al. 2010; Lin et al. 2010). A recent study has also shown that a population of $\mathrm{CD} 11 \mathrm{~b}^{+} \mathrm{Gr}-$ $1^{+}$myeloid cells from the innate immune system protect a fraction of proliferating Pten-null mouse prostate tumors from senescence, thus sustaining tumor growth (Di Mitri et al. 2014). Therefore, it remains to be further determined in what other genetic makeups prosenescence can be engaged, and what types of targeted therapies and/or immune therapies can be used to enhance cellular senescence on the one end, and to morph it into full-blown apoptosis on the other end.

\section{Cellular Metabolism}

Cancer cells are known to undergo metabolic reprogramming to support their rapid proliferation and growth. It is now well known that metabolic reprogramming is a hallmark of cancer cells (Hanahan and Weinberg 2011). Unlike other solid tumors, the majority of PCa does not show classical aerobic glycolysis; rather, de novo 
fatty acid and cholesterol synthesis is the hallmark of human PCa (Zadra et al. 2013). Many lipogenic enzymes are found deregulated in $\mathrm{PCa}$, such as fatty acid synthase, acetyl-CoAcarboxylase, and HMG-CoA reductase (Zadra et al. 2013). Additionally, it is widely postulated that a high-fat/cholesterol Western diet can promote PCa progression, and contributes to the aggressiveness of disease (Gronberg 2003; Yang et al. 2015), in support of the notion that the metabolism of lipids, whether exogenous or endogenously produced, contributes to prostate tumorigenesis and its progression. Importantly, these lipogenic enzymes can be suppressed/inhibited by small pharmacological molecules and hence represent ideal therapeutic targets. Indeed, this metabolic dimension has been explored in preclinical studies (Kridel et al. 2004; Zadra et al. 2014) and should be now investigated coclinically.

\section{CONCLUDING REMARKS}

In conclusion, the coclinical trial approach provides multiple new opportunities and tools to accelerate and facilitate the entire process of drug testing toward accelerated drug positioning and approval for precise and personalized medicine. It allows researchers to disentangle the daunting clinical complexity of cancer. In light of the potential of immunotherapy to fulfill the promise of personalized cancer treatment, future efforts will also focus on the immune system in mice, the tumor microenvironment, and how they are impacted by the genetic makeup of the GEMMs used. Integration of preclinical with clinical data in real time, which represents the cornerstone of the coclinical trial platform, promises to quickly yield important insights into issues of resistance and sensitivity, and can provide predictive guidance for both the optimization of concurrent human clinical trials and the correct interpretation of trial results. It is important to note that a specific characteristic that enables the coclinical trial approach to be so effective is its power to identify the mechanisms of tumor resistance in humans (either de novo or acquired), a common hurdle that affects the vast majority of single-agent targeted therapies and quickly tests the efficacy of new drug combinations to overcome such resistance.

With the evolving landscape of cancer research and therapy for mCRPC along with our unprecedented ability to model cancer in the mouse, further bolstered by the advent of CRISPER-Cas9 technology (Hsu et al. 2014), coclinical trials will greatly help in testing novel treatments. Many oncogenic pathways involved in cell survival, in addition to the AR pathway, are actionable and can be suppressed/inhibited by current available inhibitors. Immune therapies are also coming of age. There is little doubt that, in the years to come, the coclinical trial platform and the concept of the mouse hospital will be critical in designing new personalized combinations toward the prevention and effective treatment of mCRPC.

\section{ACKNOWLEDGMENTS}

We thank current members of the Pandolfi Laboratory for critical discussion and Lauren Southwood and Elizabeth Stack for insightful editing. This work is supported by funding from the National Cancer Institute (R35CA197529 to P. P.P.) and Department of Defense PCRP Postdoctoral Training award (W81XWH-12-10040 to M.C.).

\section{REFERENCES}

* Reference is also in this collection.

Abate-Shen C, Pandolfi PP. 2013. Effective utilization and appropriate selection of genetically engineered mouse models for translational integration of mouse and human trials. Cold Spring Harb Protoc doi: 10.1101/pdb. top078774.

Alimonti A, Nardella C, Chen Z, Clohessy JG, Carracedo A, Trotman LC, Cheng K, Varmeh S, Kozma SC, Thomas G, et al. 2010. A novel type of cellular senescence that can be enhanced in mouse models and human tumor xenografts to suppress prostate tumorigenesis. J Clin Invest 120: 681693.

Barbieri CE, Baca SC, Lawrence MS, Demichelis F, Blattner M, Theurillat JP, White TA, Stojanov P, Van Allen E, Stransky N, et al. 2012. Exome sequencing identifies recurrent SPOP, FOXA1 and MED12 mutations in prostate cancer. Nat Genet 44: 685-689.

Beer TM, Armstrong AJ, Rathkopf DE, Loriot Y, Sternberg $\mathrm{CN}$, Higano CS, Iversen P, Bhattacharya S, Carles J, Chowdhury S, et al. 2014. Enzalutamide in metastatic 
prostate cancer before chemotherapy. N Engl J Med 371: 424-433.

Beltran H, Yelensky R, Frampton GM, Park K, Downing SR, MacDonald TY, Jarosz M, Lipson D, Tagawa ST, Nanus DM, et al. 2013. Targeted next-generation sequencing of advanced prostate cancer identifies potential therapeutic targets and disease heterogeneity. Eur Urol 63: 920-926.

Boelens MC, Wu TJ, Nabet BY, Xu B, Qiu Y, Yoon T, Azzam DJ, Twyman-Saint Victor C, Wiemann BZ, Ishwaran H, et al. 2014. Exosome transfer from stromal to breast cancer cells regulates therapy resistance pathways. Cell 159: 499-513.

Boutros PC, Fraser M, Harding NJ, de Borja R, Trudel D, Lalonde E, Meng A, Hennings- Yeomans PH, McPherson A, Sabelnykova VY, et al. 2015. Spatial genomic heterogeneity within localized, multifocal prostate cancer. Nat Genet 47: 736-745.

Boysen G, Barbieri CE, Prandi D, Blattner M, Chae SS, Dahija A, Nataraj S, Huang D, Marotz C, Xu L, et al. 2015. SPOP mutation leads to genomic instability in prostate cancer. eLife 4: e09207.

Brenner JC, Ateeq B, Li Y, Yocum AK, Cao Q, Asangani IA, Patel S, Wang X, Liang H, Yu J, et al. 2011. Mechanistic rationale for inhibition of poly(ADP-ribose) polymerase in ETS gene fusion-positive prostate cancer. Cancer Cell 19: 664-678.

Carver BS, Chapinski C, Wongvipat J, Hieronymus H, Chen Y, Chandarlapaty S, Arora VK, Le C, Koutcher J, Scher H, et al. 2011. Reciprocal feedback regulation of PI3K and androgen receptor signaling in PTEN-deficient prostate cancer. Cancer Cell 19: 575-586.

* Centenera MM, Selth LA, Ebrahimie E, Butler LM, Tilley WD. 2017. New opportunities for targeting the androgen receptor in prostate cancer. Cold Spring Harb Perspect Med doi: 10.1101/cshperspect.a030478.

Chandarlapaty S. 2012. Negative feedback and adaptive resistance to the targeted therapy of cancer. Cancer Discov 2: 311-319.

Chen CD, Welsbie DS, Tran C, Baek SH, Chen R, Vessella R, Rosenfeld MG, Sawyers CL. 2004. Molecular determinants of resistance to antiandrogen therapy. Nat Med 10: 33-39.

Chen Z, Trotman LC, Shaffer D, Lin HK, Dotan ZA, Niki M, Koutcher JA, Scher HI, Ludwig T, Gerald W, et al. 2005. Crucial role of p53-dependent cellular senescence in suppression of Pten-deficient tumorigenesis. Nature 436: 725-730.

Chen Z, Cheng K, Walton Z, Wang Y, Ebi H, Shimamura T, Liu Y, Tupper T, Ouyang J, Li J, et al. 2012. A murine lung cancer co-clinical trial identifies genetic modifiers of therapeutic response. Nature 483: 613-617.

Chen Z, Akbay E, Mikse O, Tupper T, Cheng K, Wang Y, Tan X, Altabef A, Woo SA, Chen L, et al. 2014. Co-clinical trials demonstrate superiority of crizotinib to chemotherapy in ALK-rearranged non-small cell lung cancer and predict strategies to overcome resistance. Clin Cancer Res 20: 1204-1211.

Collins FS, Varmus H. 2015. A new initiative on precision medicine. $N$ Engl J Med 372: 793-795.

Comstock CE, Augello MA, Goodwin JF, de Leeuw R, Schiewer MJ, Ostrander WF Jr, Burkhart RA, McClendon AK, McCue PA, Trabulsi EJ, et al. 2013. Targeting cell cycle and hormone receptor pathways in cancer. Oncogene 32: 5481-5491.

Cooper CS, Eeles R, Wedge DC, Van Loo P, Gundem G, Alexandrov LB, Kremeyer B, Butler A, Lynch AG, Camacho N, et al. 2015. Analysis of the genetic phylogeny of multifocal prostate cancer identifies multiple independent clonal expansions in neoplastic and morphologically normal prostate tissue. Nat Genet 47: 367-372.

Di Mitri D, Toso A, Chen JJ, Sarti M, Pinton S, Jost TR, D'Antuono R, Montani E, Garcia-Escudero R, Guccini I, et al. 2014. Tumour-infiltrating Gr- $1^{+}$myeloid cells antagonize senescence in cancer. Nature 515: 134-137.

Floc'h N, Kinkade CW, Kobayashi T, Aytes A, Lefebvre C, Mitrofanova A, Cardiff RD, Califano A, Shen MM, AbateShen C. 2012. Dual targeting of the Akt/mTOR signaling pathway inhibits castration-resistant prostate cancer in a genetically engineered mouse model. Cancer Res 72: 4483-4493.

Gao H, Ouyang X, Banach-Petrosky WA, Gerald WL, Shen MM, Abate-Shen C. 2006. Combinatorial activities of Akt and B-Raf/Erk signaling in a mouse model of androgenindependent prostate cancer. Proc Natl Acad Sci 103: 14477-14482.

Gonzalez-Billalabeitia E, Seitzer N, Song SJ, Song MS, Patnaik A, Liu XS, Epping MT, Papa A, Hobbs RM, Chen M, et al. 2014. Vulnerabilities of PTEN-TP53-deficient prostate cancers to compound PARP-PI3K inhibition. Cancer Discov 4: 896-904.

Grasso CS, Wu YM, Robinson DR, Cao X, Dhanasekaran SM, Khan AP, Quist MJ, Jing X, Lonigro RJ, Brenner JC, et al. 2012. The mutational landscape of lethal castrationresistant prostate cancer. Nature 487: 239-243.

Gronberg H. 2003. Prostate cancer epidemiology. Lancet 361: 859-864.

Gundem G, Van Loo P, Kremeyer B, Alexandrov LB, Tubio JM, Papaemmanuil E, Brewer DS, Kallio HM, Hognas G, Annala M, et al. 2015. The evolutionary history of lethal metastatic prostate cancer. Nature 520: 353-357.

Hanahan D, Weinberg RA. 2011. Hallmarks of cancer: The next generation. Cell 144: 646-674.

Hsu PD, Lander ES, Zhang F. 2014. Development and applications of CRISPR-Cas9 for genome engineering. Cell 157: 1262-1278.

Huggins C, Hodges C. 1941. Studies on prostatic cancer II: The effects of castration on advanced carcinoma of the prostate gland. Arch Surg 43: 209-223.

Irshad S, Abate-Shen C. 2013. Modeling prostate cancer in mice: Something old, something new, something premalignant, something metastatic. Cancer Metastasis Rev 32: 109-122.

Ittmann M, Huang J, Radaelli E, Martin P, Signoretti S, Sullivan R, Simons BW, Ward JM, Robinson BD, Chu GC, et al. 2013. Animal models of human prostate cancer: The consensus report of the New York Meeting of the Mouse Models of Human Cancers Consortium Prostate Pathology Committee. Cancer Res 73: 2718-2736.

Kantoff PW, Higano CS, Shore ND, Berger ER, Small EJ, Penson DF, Redfern CH, Ferrari AC, Dreicer R, Sims RB, et al. 2010. Sipuleucel-T immunotherapy for castration-resistant prostate cancer. N Engl J Med 363: 411-422. 
Kinkade CW, Castillo-Martin M, Puzio-Kuter A, Yan J, Foster TH, Gao H, Sun Y, Ouyang X, Gerald WL, CordonCardo C, et al. 2008. Targeting AKT/mTOR and ERK MAPK signaling inhibits hormone-refractory prostate cancer in a preclinical mouse model. J Clin Invest 118: 3051-3064.

Kridel SJ, Axelrod F, Rozenkrantz N, Smith JW. 2004. Orlistat is a novel inhibitor of fatty acid synthase with antitumor activity. Cancer Res 64: 2070-2075.

Kwong LN, Boland GM, Frederick DT, Helms TL, Akid AT, Miller JP, Jiang S, Cooper ZA, Song X, Seth S, et al. 2015 Co-clinical assessment identifies patterns of BRAF inhibitor resistance in melanoma. J Clin Invest 125: 1459-1470.

Lin HK, Chen Z, Wang G, Nardella C, Lee SW, Chan CH, Yang WL, Wang J, Egia A, Nakayama KI, et al. 2010. Skp2 targeting suppresses tumorigenesis by Arf-p53- independent cellular senescence. Nature 464: 374-379.

Liu J, Pan S, Hsieh MH, Ng N, Sun F, Wang T, Kasibhatla S, Schuller AG, Li AG, Cheng D, et al. 2013. Targeting Wntdriven cancer through the inhibition of Porcupine by LGK974. Proc Natl Acad Sci 110: 20224-20229.

Lunardi A, Ala U, Epping MT, Salmena L, Clohessy JG, Webster KA, Wang G, Mazzucchelli R, Bianconi M, Stack EC, et al. 2013. A co-clinical approach identifies mechanisms and potential therapies for androgen deprivation resistance in prostate cancer. Nat Genet 45: 747-755.

Ma F, Ye H, He HH, Gerrin SJ, Chen S, Tanenbaum BA, Cai C, Sowalsky AG, He L, Wang H, et al. 2016. SOX9 drives WNT pathway activation in prostate cancer. J Clin Invest 126: $1745-1758$.

Mateo J, Carreira S, Sandhu S, Miranda S, Mossop H, PerezLopez R, Nava Rodrigues D, Robinson D, Omlin A, Tunariu N, et al. 2015. DNA-repair defects and olaparib in metastatic prostate cancer. N Engl J Med 373: 1697-1708.

Nardella C, Lunardi A, Patnaik A, Cantley LC, Pandolfi PP. 2011. The APL paradigm and the "co-clinical trial" project. Cancer Discov 1: 108-116.

Palanisamy N, Ateeq B, Kalyana-Sundaram S, Pflueger D, Ramnarayanan K, Shankar S, Han B, Cao Q, Cao X, Suleman K, et al. 2010. Rearrangements of the RAF kinase pathway in prostate cancer, gastric cancer and melanoma. Nat Med 16: 793-798.

* Risbridger G. 2017. Xenografts, organoids, and other explant models. Cold Spring Harb Perspect Med doi: 10.1101/ cshperspect.a030536.

Robinson D, Van Allen EM, Wu YM, Schultz N, Lonigro RJ, Mosquera JM, Montgomery B, Taplin ME, Pritchard CC, Attard G, et al. 2015. Integrative clinical genomics of advanced prostate cancer. Cell 161: 1215-1228.

Ryan CJ, Smith MR, de Bono JS, Molina A, Logothetis CJ, de Souza P, Fizazi K, Mainwaring P, Piulats JM, Ng S, et al. 2013. Abiraterone in metastatic prostate cancer without previous chemotherapy. N Engl J Med 368: 138-148.

Scher HI, Fizazi K, Saad F, Taplin ME, Sternberg CN, Miller K, de Wit R, Mulders P, Chi KN, Shore ND, et al. 2012. Increased survival with enzalutamide in prostate cancer after chemotherapy. N Engl J Med 367: 1187-1197.

Schwartz S, Wongvipat J, Trigwell CB, Hancox U, Carver BS, Rodrik-Outmezguine V, Will M, Yellen P, de Stanchina E,
Pre- and Coclinical Studies in Prostate Cancer

Baselga J, et al. 2015. Feedback suppression of PI3Ko signaling in PTEN-mutated tumors is relieved by selective inhibition of PI3K $\beta$. Cancer Cell 27: 109-122.

Sharpless NE, Depinho RA. 2006. The mighty mouse: Genetically engineered mouse models in cancer drug development. Nat Rev Drug Discov 5: 741-754.

Storm EE, Durinck S, de Sousa e Melo F, Tremayne J, Kljavin N, Tan C, Ye X, Chiu C, Pham T, Hongo JA, et al. 2016. Targeting PTPRK-RSPO3 colon tumours promotes differentiation and loss of stem-cell function. Nature 529: 97-100.

Takahashi A, Ohtani N, Yamakoshi K, Iida S, Tahara H, Nakayama K, Nakayama KI, Ide T, Saya H, Hara E. 2006. Mitogenic signalling and the p16INK4a-Rb pathway cooperate to enforce irreversible cellular senescence. Nat Cell Biol 8: 1291-1297.

Taylor BS, Schultz N, Hieronymus H, Gopalan A, Xiao Y, Carver BS, Arora VK, Kaushik P, Cerami E, Reva B, et al. 2010. Integrative genomic profiling of human prostate cancer. Cancer Cell 18: 11-22.

Toren P, Kim S, Johnson F, Zoubeidi A. 2016. Combined AKT and MEK pathway blockade in pre-clinical models of enzalutamide-resistant prostate cancer. PloS ONE 11: e0152861.

Van Dyke T, Jacks T. 2002. Cancer modeling in the modern era: Progress and challenges. Cell 108: 135-144.

Wang XS, Shankar S, Dhanasekaran SM, Ateeq B, Sasaki AT, Jing X, Robinson D, Cao Q, Prensner JR, Yocum AK, et al 2011. Characterization of KRAS rearrangements in metastatic prostate cancer. Cancer Discov 1: 35-43.

Watson PA, Arora VK, Sawyers CL. 2015. Emerging mechanisms of resistance to androgen receptor inhibitors in prostate cancer. Nat Rev Cancer 15: 701-711.

Whitley MJ, Cardona DM, Lazarides AL, Spasojevic I, Ferrer JM, Cahill J, Lee CL, Snuderl M, Blazer DG III, Hwang ES, et al. 2016. A mouse-human phase 1 co-clinical trial of a protease-activated fluorescent probe for imaging cancer. Sci Transl Med 8: 320ra324.

Wu JN, Fish KM, Evans CP, Devere White RW, Dall'Era MA. 2014. No improvement noted in overall or causespecific survival for men presenting with metastatic prostate cancer over a 20-year period. Cancer 120: 818-823.

Yamamoto Y, De Velasco MA, Kura Y, Nozawa M, Hatanaka Y, Oki T, Ozeki T, Shimizu N, Minami T, Yoshimura K, et al. 2015. Evaluation of in vivo responses of sorafenib therapy in a preclinical mouse model of PTEN-deficient of prostate cancer. J Transl Med 13: 150.

Yang M, Kenfield SA, Van Blarigan EL, Batista JL, Sesso HD, Ma J, Stampfer MJ, Chavarro JE. 2015. Cancer Prev Res (Phila) 8: 545-551.

Zadra G, Photopoulos C, Loda M. 2013. The fat side of prostate cancer. Biochim Biophys Acta 1831: 1518-1532.

Zadra G, Photopoulos C, Tyekucheva S, Heidari P, Weng QP, Fedele G, Liu H, Scaglia N, Priolo C, Sicinska E, et al. 2014. A novel direct activator of AMPK inhibits prostate cancer growth by blocking lipogenesis. EMBO Mol Med 6: $519-538$. 


\title{
$\&_{\mathrm{CSH}}^{\infty} \&$ Cold Spring Harbor

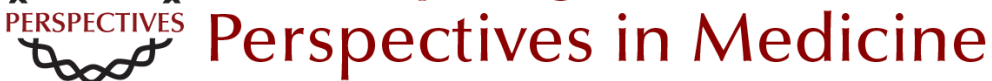

\section{Preclinical and Coclinical Studies in Prostate Cancer}

\author{
Ming Chen and Pier Paolo Pandolfi
}

Cold Spring Harb Perspect Med 2018; doi: 10.1101/cshperspect.a030544 originally published online October 16, 2017

\section{Subject Collection Prostate Cancer}

Anatomic and Molecular Imaging in Prostate Cancer

Eric T. Miller, Amirali Salmasi and Robert E. Reiter

The Epidemiology of Prostate Cancer Claire H. Pernar, Ericka M. Ebot, Kathryn M. Wilson, et al.

Prostate Stem Cells and Cancer Stem Cells Jia J. Li and Michael M. Shen

Prostate Cancer Epigenetics: From Basic Mechanisms to Clinical Implications Srinivasan Yegnasubramanian, Angelo M. De Marzo and William G. Nelson

\section{The Genomics of Prostate Cancer: A Historic} Perspective Mark A. Rubin and Francesca Demichelis

Neuroendocrine Differentiation in Prostate

Cancer: Emerging Biology, Models, and Therapies Loredana Puca, Panagiotis J. Vlachostergios and Himisha Beltran

DNA Damage Response in Prostate Cancer Matthew J. Schiewer and Karen E. Knudsen

Transcriptional Regulation in Prostate Cancer David P. Labbé and Myles Brown
New Opportunities for Targeting the Androgen Receptor in Prostate Cancer Margaret M. Centenera, Luke A. Selth, Esmaeil Ebrahimie, et al.

Prostate Cancer Research at the Crossroads Michael M. Shen and Mark A. Rubin

Immunotherapy for Prostate Cancer Nicholas J. Venturini and Charles G. Drake

Molecular Pathology of High-Grade Prostatic Intraepithelial Neoplasia: Challenges and Opportunities Levent Trabzonlu, Ibrahim Kulac, Qizhi Zheng, et al.

Metastases in Prostate Cancer Federico La Manna, Sofia Karkampouna, Eugenio Zoni, et al.

Genetically Engineered Mouse Models of Prostate Cancer in the Postgenomic Era Juan M. Arriaga and Cory Abate-Shen

Molecular Biomarkers in the Clinical Management of Prostate Cancer Aaron M. Udager and Scott A. Tomlins

Metabolic Vulnerabilities of Prostate Cancer: Diagnostic and Therapeutic Opportunities Giorgia Zadra and Massimo Loda

For additional articles in this collection, see http://perspectivesinmedicine.cshlp.org/cgi/collection/ 